\title{
Editorial
}

\section{In search of a shared language in neuropsychology}

\author{
Roberto Cubelli ${ }^{a, *}$ and Sergio Della Sala ${ }^{b}$ \\ a Department of Psychology and Cognitive Sciences, University of Trento, Italy \\ ${ }^{\mathrm{b}}$ Human Cognitive Neuroscience, Psychology, University of Edinburgh, Edinburgh, UK
}

\section{A R T I C L E I N F O}

Article history:

Received 13 March 2017

Accepted 14 March 2017

Published online 29 March 2017

We demand rigidly defined areas of doubt and uncertainty! Douglas Adams, The Hitchhiker's Guide to the Galaxy

Chi parla male, pensa male e vive male. Bisogna trovare le parole giuste: le parole sono importanti! (Who speaks poorly, thinks poorly and lives poorly. We ought to find the right words: words are important!)

Nanni Moretti, Bianca (movie), 1989

Definitions provide a shared knowledge and a common understanding of a topic. Agreed definitions are necessary for communication and interconnected activities. This is relevant when talking, writing or reading about science.

Definitions can serve a variety of different functions, which are not mutually exclusive: nominal, taxonomic, descriptive and explicative. The meaning of a noun should allow all speakers and users of the same language to represent and think of the same object in the same way. By providing either an unambiguous description of the intended phenomenon or a grounded interpretation of its underlying causes, each definition should permit a clear identification and classification of the referent objects. Such lucidity in the use of language is a joy (Hoffman, 1989). In neuropsychology, as well as in all other scientific domains, definitions should suggest clear labels and classifications, but should avoid explanations and avert referencing to specific theories or models to prevent failures in communication or hurdles to further investigations and discoveries.

The label "semantic paraphasia" designs errors in spontaneous speech and oral naming, in which the intended word is substituted by a semantically related word. This definition refers to a well-defined category of errors, which does not include either spelling and reading errors (labeled paragraphias and paralexias, respectively) or errors with a different target-response relationship (e.g., formal similarity). It is well known that semantic errors can arise from different loci of cognitive impairment and reflect different processing levels (Caramazza \& Hillis, 1990). However, this definition is theoretically neutral, as it does not suggest any account of the underlying causes and mechanisms.

Incomplete, ambiguous or idiosyncratic definitions can preclude communication, thus harming thinking and the possibility to detect unexpected phenomena or to explore new topics. Here are three examples illustrating the problems deriving from the lack of precise definitions.

(i) One the most popular definitions of unilateral spatial neglect states that it is "a failure to report, respond, or orient to contralateral stimuli that is not caused by an elemental sensorimotor deficit" (Heilman, Valenstein, \& Watson, 2000). This definition encompasses all the negative signs of neglect, but disregards productive phenomena, such as allochiria (Vallar, Zilli, Gandola, \& Bottini, 2006), as they were then not related to neglect.

(ii) Phonemic paraphasias are usually defined as errors involving "the reordering, omission, or addition of correctly pronounced phonemes" (Shallice, 1988). According to this definition, the erroneous response, i.e., the resulting phonemic sequence, can be either a word (e.g., dog $\rightarrow$ fog) or a non-word (e.g., dog $\rightarrow$ vog), indifferently. Nevertheless, some authors (for instance

\footnotetext{
* Corresponding author. Department of Psychology and Cognitive Sciences, University of Trento, Italy.

E-mail addresses: roberto.cubelli@unitn.it (R. Cubelli), sergio@ed.ac.uk (S. Della Sala). 
Lesser, 1978), called phonemic paraphasia only the nonword responses in which the target word is identifiable. When the response is a phonologically related word, (i.e., with similar form but not similar meaning), the error is assigned to a different category and called "formal verbal paraphasia". Lecours, Lhermitte, and Bryans (1983) maintained that "verbal paraphasias of a formal type constitute a particular category of phonemic paraphasias" (p. 43), but errors limited to formal paraphasias have been described in individual patients (e.g., Blanken, 1990), suggesting that word responses may reflect different impaired mechanisms. The uncertainty in classifying wrong responses into alternative classes can lead to errors in interpretation and diagnosis.

(iii) Traditionally, limb apraxia comprises ideational and ideomotor forms. This distinction has different meanings for different authors: for Morlaas (1928) it distinguishes different gestures types (transitive and intransitive), for Hécaen (1972) different levels of gestures complexity (complex and simple), for De Renzi (1985) different tasks (production on command and imitation). The same label is hence used to refer to different clinical performances.

We need to discuss the referential function of names and to acquire a clearer view of our use of labels and definitions. This endeavour is independent from the theoretical models proposed to account for observed phenomena. Following Locke (1690), who introduced the notion of "nominal essence" as the "abstract Idea to which the Name is annexed", we should address the issue of nominal essence or nominal definition in neuropsychology.

From this volume, within the Discussion Forum section of Cortex, we plan to publish definitions of terms used in neuropsychology parlance. Each entry will aim at describing a term used in neuropsychology listing the distinctive features but ignoring the associated characteristics which may be important clinically or scientifically, yet do not stipulate a definition. Typically, neuroanatomical localizations are significant but they do not necessarily serve to define a term.

A definition should be different from a brief entry in an encyclopaedia or a very brief review. It is an explicit and strict assertion, with no examples or ambiguous terms, allowing the precise identification of the target clinical picture, sign or behaviour. Further, since neuropsychological deficits may show multifarious phenomena, a definition should state that $\mathrm{x}$ or $\mathrm{y}$ are possible features of a given symptom or syndrome, not that they always present with $\mathrm{x}$ or $\mathrm{y}$. A definition should aim at the best common denominator offering a term, allowing everybody using it to understand the same thing. A definition is the best possible relationship between a name and a thing.

Each definition will be the result of a discussion among experts to reach a common denominator agreed by most if not all. This procedure aims at excluding from each definition, controversial aspects of the entries, personal takes or conflicting hypotheses. Definitions will therefore be conservative and subject to change should new, pertinent evidence emerge.

We accept that these definitional attempts will not be perfect, but hope that they would go a long way in avoiding the use of implicit definitions conducive of misunderstanding and confusion. It is inevitable that some of you will enthusiastically consent with this initiative, whereas others will be more critical. Anybody willing can take part. Please submit proposals or preliminary drafts for entries to the Cortex office (cortex@ed.ac.uk). Definitions can apply to syndromes, symptoms, signs, paradigms, procedures or neuroanatomy relevant to neuropsychology. When sending a proposal for a definition, please suggest a short list of colleagues who may help in refining this definition, until consensus is reached.

\section{R E F E R E N C E S}

Blanken, G. (1990). Formal paraphasias: A single case study. Brain and Language, 38(4), 534-554.

Caramazza, A., \& Hillis, A. E. (1990). Where do semantic errors come from? Cortex, 26(1), 95-122.

De Renzi, E. (1985). Methods of limb apraxia examination and their bearing on the interpretation of the disorder. In E. Roy (Ed.), Neuropsychological studies of apraxia and related disorders (pp. 45-64). Amsterdam: Elsevier, North Holland.

Hécaen, H. (1972). Introduction à la neuropsychologie. Paris: Larousse.

Heilman, K. M., Valenstein, E., \& Watson, R. T. (2000). Neglect and related disorders. Seminars in Neurology, 20(4), 463-470.

Hoffman, E. (1989). Lost in translation. New York: Penguin Books.

Lecours, A. R., Lhermitte, F., \& Bryans, B. (1983). Aphasiology. London: Bailliere-Tindall.

Lesser, R. (1978). Linguistic investigations of aphasia. London: Arnold.

Locke, J. (1690). Essay concerning human understanding. London: Thomas Bassett.

Morlaas, J. (1928). Contribution à l'étude de l'apraxie. Paris: Legrand.

Shallice, T. (1988). From neuropsychology to mental structure. Cambridge: Cambridge University Press.

Vallar, G., Zilli, T., Gandola, M., \& Bottini, G. (2006). Productive and defective impairments in the neglect syndrome: Graphic perseveration, drawing productions and optic prism exposure. Cortex, 42(6), 911-920. 